\title{
Positron Annihilation in Steel Burnished by Vibratory Shot Peening
}

\author{
R. ZALESKI ${ }^{a, *}$ AND K. ZALESKI ${ }^{b}$ \\ ${ }^{a}$ Institute of Physics, Maria Curie-Skłodowska University \\ pl. Marii Curie-Skłodowskiej 1, 20-031 Lublin, Poland \\ ${ }^{b}$ Faculty of Mechanical Engineering, Lublin University of Technology \\ Nadbystrzycka 36, 20-618 Lublin, Poland

\begin{abstract}
A series of C45 steel samples was burnished by shot peening with varying time of treatment. The samples were investigated by nondestructive positron annihilation techniques: angular correlations of two-quantum annihilation radiation and positron annihilation lifetime spectroscopy. To determine residual stresses present under burnished surface the same samples were studied by destructive Davidenkov method. Change of absolute value of the weighted average of residual stresses over positron range in the series of the samples is in good agreement with change of $S / W$ ratio obtained by angular correlations of two-quantum annihilation radiation. Both parameters increase during first 10 minutes of shot peening and then stabilize. Positron annihilation lifetime spectra allowed to identify two types of structural defects: smaller ones like vacancies or dislocations and bigger - probably clusters of vacancies. Increase in shot peening time causes reduction of positron trapping rate and lifetime rise in bigger defects.
\end{abstract}

PACS numbers: 78.70.Bj, 71.60.+z, 62.20.Fe, 62.20.Mk, 81.20.Wk, 81.40.- $\mathrm{z}, 81.70 .-\mathrm{q}, 81.05 . \mathrm{Bx}$

\section{Introduction}

Steel elements of machines are often put under changing load. Therefore their important property is the fatigue strength. Increase in the fatigue strength can be obtained by burnishing [1]. It results in plastic deformation of the surface layer as a consequence of burnishing tool impact. The most important effects of burnishing on surface layer are: material density decrease, increase in hardness, change of surface roughness, and appearance of compressive stresses below the surface. Consolidation of the top layer of the material is explained by the theory of dislocations [2]. There is a distinct growth in density of dislocations after

*corresponding author; e-mail: radek.zaleski@umcs.lublin.pl 
burnishing process. Standard methods of characterization of burnished surface, like measurements of micro- and mezohardness and residual stresses, are usually destructive. It was already shown (e.g. [3, 4]) that nondestructive positron annihilation methods provide information consistent with results of mechanical methods in the case of metals burnished by cold rolling. Usually metals of high purity (e.g. iron [5]) were investigated and there is scant information concerning steel. Moreover there are very few papers concerning influence of other burnishing methods on positron annihilation results.

One of the burnishing methods that was not studied by positron annihilation technique so far is shot peening [1]. Contrary to the cold rolling, shot peening is characterized by dynamic reaction of the peening medium (usually balls) on the surface. The advantage of this method is its ability to burnish complicated shapes and elements having low rigidity. Like other methods of burnishing, the shot peening causes formation of compressive stresses in the surface layer of the material [6]. The scale of changes depends on burnishing conditions (e.g. frequency and amplitude of burnishing tool movement, shape of peening medium, time of burnishing). As the residual stresses presence is related to changes of structural defect density in the material (usually dislocations), one may anticipate that defect sensitive positron annihilation techniques are suitable to estimate the fatigue strength changes caused by shot peening. This is already confirmed by our preliminary results obtained by angular correlations of two-quantum annihilation radiation (ACAR) [7].

In this work the unalloyed $\mathrm{C} 45$ steel burnished by shot peening was investigated. Series of steel samples was prepared in the Lublin University of Technology. The only varied parameter of burnishing in this series was shot peening time. Two techniques involving positron annihilation were used: ACAR and positron annihilation lifetime spectroscopy (PALS). The results were compared to mechanical measurements of residual stresses.

\section{Experimental}

The samples studied were $\mathrm{C} 45$ steel bars $\left(4 \times 15 \times 100 \mathrm{~mm}^{3}\right)$ of chemical composition presented in Table. Recrystallizing and stress relief annealing was performed on the samples before burnishing. The surface of the samples was initially ground. The samples were fastened to the bottom of shot peening machine chamber. The chamber was partially filled by steel ball bearing balls (diameter $6 \mathrm{~mm}$ ) serving as peening medium. The burnishing was performed by setting

TABLE

Chemical composition of unalloyed steel C45 samples.

\begin{tabular}{c|c|c|c|c|c|c|c|c|c}
\hline \hline Element & $\mathrm{Fe}$ & $\mathrm{Mn}$ & $\mathrm{C}$ & $\mathrm{Ni}$ & $\mathrm{Si}$ & $\mathrm{Cr}$ & $\mathrm{Cu}$ & $\mathrm{S}$ & $\mathrm{P}$ \\
\hline Mass fraction [\%] & 98.28 & 0.55 & 0.44 & 0.25 & 0.21 & 0.16 & 0.08 & 0.02 & 0.01
\end{tabular}


the chamber into vertical vibratory motion with amplitude $58 \mathrm{~mm}$ and frequency $7 \mathrm{~Hz}$. The surface layer of the samples was strain hardened due to the impact of bouncing balls.

Residual stress measurements were performed by the Davidenkov method [8]. The successive layers of the sample were removed by chemical etching in $4 \%$ nitric acid. In order to achieve constant speed of etching, the reaction products were constantly removed from the surface by moving brush. Relation between deflection of sample $f(z)$ and thickness of removed layer was measured. Depth profile of residual stresses was calculated using the Davidenkov-Birger equation

$$
\sigma(z)=\frac{4 E}{3 l^{2}}\left[(h-z)^{2} \frac{\mathrm{d} f(z)}{\mathrm{d} z}-4(h-z) f(z)+2 \int_{0}^{z} f(\xi) \mathrm{d} \xi\right],
$$

where $E$ - the Young modulus, $l$ - the length of etched surface, $h$ - the thickness of sample before etching, $z$ - the distance from sample surface, $f(x)$ - the deflection of sample after removal of layer of thickness $x$.

Typical distribution of residual stresses in the sample burnished by shot peening is shown in Fig. 1. The range of positrons emitted from ${ }^{22} \mathrm{Na}$ in steel

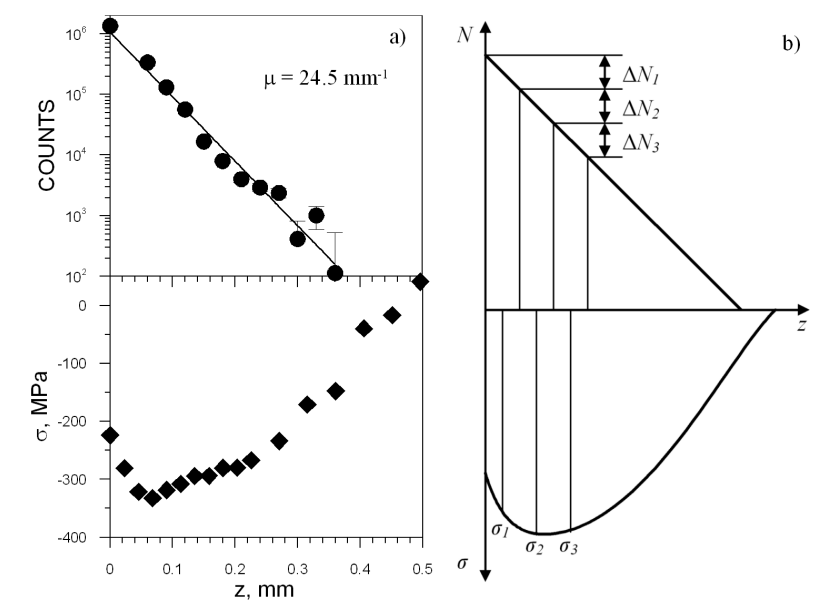

Fig. 1. (a) Range of positrons in the investigated C45 steel samples (above). Absorption coefficient $\mu$ was obtained from the fit presented as solid line. Depth profile of residual stresses for steel burnished by shot peening for 10 min (below). (b) Schematic idea of calculation of residual stress weighted average.

is smaller than the depth of residual stress distribution (Fig. 1a). The range of positrons was measured using a set of $0.06 \mathrm{~mm}$ and $0.09 \mathrm{~mm}$ thick steel foils. Absorption coefficient $\mu=24.5 \mathrm{~mm}^{-1}$ was obtained by fitting equation $N=$ $N_{0} \exp (-\mu d)$ to the experimental data, where $N_{0}$ is number of positrons emitted from the source and $d$ is thickness of absorbent. In order to compare the results of positron annihilation methods and stresses measurement, weighted average of residual stresses over positron range in the samples was calculated 


$$
\sigma_{\mathrm{w}}=\frac{\sum_{i} \Delta N_{i} \sigma_{i}}{\sum_{i} \Delta N_{i}},
$$

where $\sigma_{i}$ - the $i$-th value of residual stress, $\Delta N_{i}$ - the change of count number in the absorption curve in the depth range corresponding to $\sigma_{i}$ (as shown in Fig. 1b).

The angular correlations of two-quantum annihilation radiation were measured using a long-slit apparatus [9] with angular resolution of 1 mrad. The distance between the sample and scintillation counters was $2.85 \mathrm{~m}$. The source of positrons was $370 \mathrm{MBq}{ }^{22} \mathrm{Na}$ (Amersham). Over $2.5 \times 10^{5}$ coincidences per ACAR curve were collected. The parameter $S$ was defined as the number of counts within angle interval of $7 \mathrm{mrad}$ around the centre of the curve. The parameter $W$ was calculated as sum of counts for angles of range from -14 to -8 mrad and from 8 to $14 \mathrm{mrad}$. Because both $S$ and $W$ showed rather large statistical variations, the ratio $S / W$ was assumed to represent ACAR results.

Positron lifetime spectra were measured using standard fast-slow coincidence lifetime spectrometer equipped in $\mathrm{BaF}_{2}$ scintillators [10]. Time resolution was about 240 ps at FWHM. The $0.4 \mathrm{MBq}{ }^{22} \mathrm{Na}$ positron source sealed in $7.6 \mu \mathrm{m}$ thick Kapton envelope was placed between two identical samples; $10^{7}$ counts were collected per spectrum. The PALS spectra were analysed using the LT programme [11]. The model function used by LT was assumed to consist of two exponential components convoluted with the resolution curve (Gauss function) plus the constant random coincidence background. Correction for positron absorption in the source envelope was assumed to be $12 \%$ with lifetime 374 ps (after [12]).

\section{Results and discussion}

Modification of the C45 steel surface burnished by shot peening is clearly visible by eye - roughness of the surface changes, sample thickness reduces and sample deflection rises with increase in burnishing time, indicating appearance of residual stresses. The change of the weighted average of residual stresses $\sigma_{\mathrm{w}}$ after shot peening time confirms this observation (Fig. 2). Initially, in annealed and ground sample residual stresses are close to zero. During first 10 minutes of vibrational burnishing $\sigma_{\mathrm{w}}$ decreases. It stabilizes on about $-280 \mathrm{MPa}$ when the time of shot peening is longer than 10 minutes. Negative values of $\sigma_{\mathrm{w}}$ indicate compressive stresses.

Similar tendency of $\sigma_{\mathrm{w}}$ change with shot peening time was observed in the case of $S / W$ parameter ratio (Fig. 2). The increase in $S / W$ is about $10 \%$ indicating narrowing of angular correlation curve. Usual interpretation of such results is increase in the contribution of positrons annihilating with conduction electrons which have small momenta [13]. It may point out that the fraction of positrons trapped in the defects of crystalline structure, like vacancies and dislocations, raises. As in the case of the weighted average of residual stresses, the values of $S / W$ increase with shot peening time only for the samples burnished for less than 10 minutes. An agreement between $\sigma_{\mathrm{w}}$ and $S / W$ confirms that residual stresses 


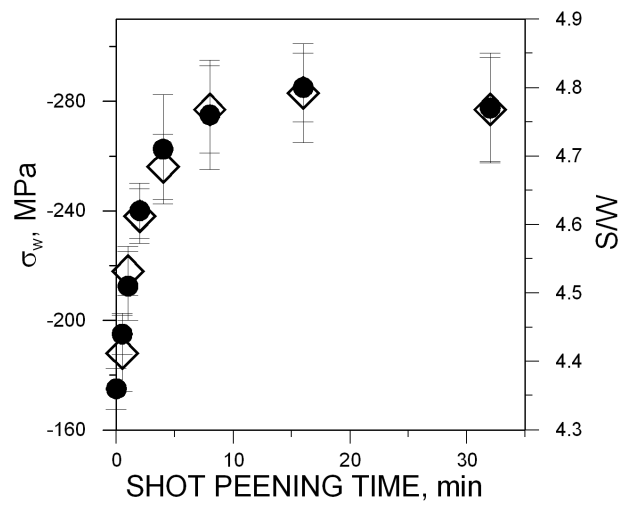

Fig. 2. Values of $S / W$ parameter obtained by ACAR (full circles) and weighted average of residual stresses $\sigma_{\mathrm{w}}$ (open diamonds) as a function of shot peening time.

origin is an increase in defect density. Analogous rise of $S$ parameter was observed for cold rolled nickel [14]. In this case the saturation of $S$ value was explained as positron trapping in vacancies only.

Analysis of lifetime spectra parameters showed that difference of average lifetime $\tau_{\mathrm{av}}=I_{1} \tau_{1}+I_{2} \tau_{2}$ between samples is negligibly small and $\tau_{\mathrm{av}}$ cannot serve as an indicator of sample properties. Thus, separation of two components in the spectra seems necessary. Contribution of short-lived component is dominating (Fig. 3). Its lifetime $\tau_{1}=140-150 \mathrm{ps}$ corresponds to annihilation of positrons trapped in dislocations in iron [15] but also in single vacancies [16] often accompanying the dislocations. Unlike mechanical methods it is known that positron techniques are more sensitive to vacancies than to dislocations [17]. It is not surprising that in polycrystalline steel almost all positrons are trapped. Also the second component is of defect origin. Its lifetime $\tau_{2}=280-340 \mathrm{ps}$ corresponds to larger voids. Assuming that these voids are vacancy clusters [18], their size can be estimated to rise from about 5 to 10 vacancies in a cluster [19]. Intensity of the long-lived component is initially $11 \%$ but it decreases to $5 \%$ with increase in shot peening time. The intensity change is accompanied by almost linear (except non-burnished sample) increase in lifetimes.

From the results of lifetime spectra analysis the "bulk" lifetime

$$
\frac{1}{\tau_{\mathrm{b}}}=\frac{I_{1}}{\tau_{1}}+\frac{I_{2}}{\tau_{2}}
$$

and positron trapping rate $\kappa$ in the larger voids

$$
\kappa=\frac{I_{2}}{I_{1}}\left(\frac{1}{\tau_{\mathrm{b}}}-\frac{1}{\tau_{2}}\right)
$$

were determined using a two state trapping model [20]. Although the model was developed for samples where only one kind of traps is present, it was also proved useful in the case of materials with more complex structure of defects like the investigated steel. 


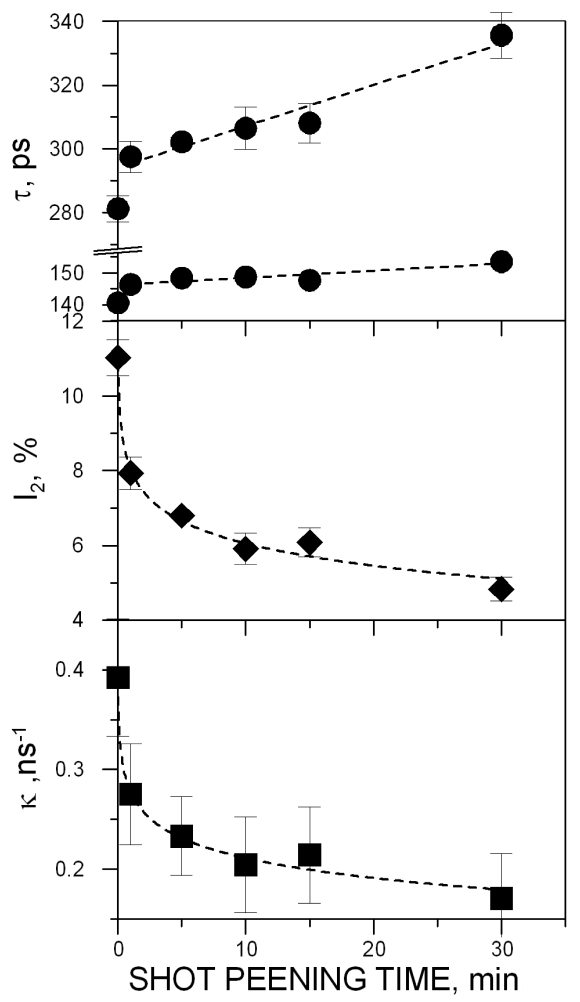

Fig. 3. Lifetimes, intensity of the long-lived component and positron trapping rate as a function of shot peening time. Dashed lines are added as eye guide.

Obtained $\kappa$ values vary from $0.39 \mathrm{~ns}^{-1}$ in non-burnished sample to $0.17 \mathrm{~ns}^{-1}$ after 30 minutes of shot peening. Fast initial decrease in positron trapping rate slows down with increasing the shot peening time resulting in the dependence similar to that observed in the case of $S / W$ ratio and weighted average of residual stresses. The PALS parameter change suggests that reorganization of defects occurs as a consequence of plastic deformations caused by shot peening. Large defects (vacancy clusters probably) group themselves into bigger structures causing increase in $\tau_{2}$ and decrease in $\kappa$. Because $\kappa=\mu_{\mathrm{D}} c$, where $\mu_{\mathrm{D}}$ is positron trapping rate specific for defect type and $c$ is concentration of defects, $\kappa$ decrease may be a consequence of both decrease in defect concentration and change of $\mu_{\mathrm{D}}$ occurring when defect type is changed. Nonzero contribution of the second effect is suggested by $\tau_{2}$ rise. The decrease in positron trapping rate in larger defects may also be a consequence of the increase in density of single vacancies and dislocations.

There is also a distinct difference in PALS results between non-burnished surface and sample that was shot peened for short (1 minute) time. It may be the result of surface roughness change. The profilogram of ground sample shows abrupt changes with about $4 \mu \mathrm{m}$ period and mean deviation from average surface 
$R_{\mathrm{a}}=1 \mu \mathrm{m}$ while surface of burnished sample has about $100 \mu \mathrm{m}$ period and $R_{\mathrm{a}}=0.7 \mu \mathrm{m}$. Such change has to reflect in the change of positron surface states that influence PALS spectra shape.

\section{Conclusions}

Changes of parameters obtained by both annihilation methods used: PALS and ACAR are subtle but it can be revealed if spectra are processed thoroughly. Large number of counts is recommended.

The rise of $S / W$ ratio obtained by the angular correlations of two-quantum annihilation radiation shows that defect density in the sample increases with extension of shot peening time if it is shorter than 10 minutes. For longer burnishing times there is no distinct change in $S / W$ that may be interpreted as positron trapping mainly in vacancies. The same type of dependence can be found when the change of weighted average of residual stresses is considered.

The PALS results prove the existence of two types of defects that may be identified as dislocations accompanied by single vacations (smaller ones) and vacancy clusters (bigger ones). If shot peening time is increased, vacancy clusters become larger which results in an increase in trapped positrons lifetime. It goes along with the decrease in positron trapping rate in bigger defects. This effect may be caused by grouping of vacancy clusters as well as increase in density of single vacancies and dislocations.

A distinct difference in macroscopic surface properties, like roughness, between non-burnished sample and shot peened one reflects in rapid change of PALS parameters. It may be caused by change of density and probably by the type of positron surface states.

\section{Acknowledgments}

Authors thank Prof. Tomasz Goworek for valuable discussions and remarks. The assistance of Dr. Wiesław Gustaw in ACAR measurements is gratefully acknowledged.

\section{References}

[1] W. Przybylski, Technology of Burnishing, WNT, Warszawa 1987, p. 127 (in Polish).

[2] D.D. Papshev, Hardening Finishing by Surface Plastic Deformation, Mashinostroenie, Moscow 1978, p. 33 (in Russian).

[3] M.S. Abd El Kerim, J. Mater. Eng. Perform. 11, 428 (2002).

[4] E. Gomaa, N. Mostafa, M. Mohsen, A.S. Taha, J. Mater. Eng. Perform. 12, 190 (2002).

[5] C.L. Snead, Jr., A. N. Goland, J. H. Kusmiss, H. C. Huang, R. Meade, Phys. Rev. $B$ 3, 275 (1971). 
[6] R. Fathallah, H. Sidhom, C. Braham, L. Castex, Mater. Sci. Technol. 19, 1050 (2003).

[7] K. Zaleski, A. Parol, W. Gustaw, T. Goworek, in: Proc. III Conf. on Technology of Processing by Burnishing, Bydgoszcz (Poland) 1984, Ed. M.M. Kozłowski, Technical-Agriculture Academy, Bydgoszcz 1984, p. 231.

[8] I.A. Birger, Residual Stresses, Mashgiz, Moscow 1963, p. 65 (in Russian).

[9] J. Wawryszczuk, C. Rybka, W. Gustaw, Fol. Societ. Sci. Lub. 16, 101 (1974).

[10] R. Zaleski, Ph.D. thesis, Maria Curie-Skłodowska University, Lublin 2005, p. 55.

[11] J. Kansy, Nucl. Instrum. Methods Phys. Res. A 374, 235 (1996).

[12] T. Goworek, W. Górniak, J. Wawryszczuk, Nucl. Instrum. Methods Phys. Res. A 321, 560 (1992).

[13] J. Dryzek, Investigation of Crystal Lattice Defects by Positron Annihilation, The Henryk Niewodniczański Institute of Nuclear Physics PAS, Kraków 1994, p. 58 (in Polish).

[14] G. Dlubek, O. Brümmer, E. Hensel, Phys. Status Solidi A 34, 737 (1976).

[15] Yong-Ki Park, J.T. Waber, M. Meshii, C.L. Snead, C.G. Park, Phys. Rev. B 34, 823 (1986).

[16] H.E. Schaefer, Phys. Status Solidi A 102, 47 (1987).

[17] E. Dryzek, Acta Phys. Pol. A 107, 753 (2005).

[18] W. Rudzińska, J. Pająk, M. Szuszkiewicz, G. Bujnarowski, A.A. Kluza, in: Proc. 34 th Polish Seminar on Positron Annihilation, Opole (Poland) 2002, Ed. K. Jerie, University of Opole, University of Wrocław, Opole 2002, p. 69.

[19] M.J. Puska, R.M. Nieminen, J. Phys. F, Met. Phys. 13, 333 (1983).

[20] W. Brandt, Appl. Phys. 5, 1 (1974). 